\title{
Standards bringen vielfältig Nutzen
}

Seit nunmehr zwei Jahren arbeite und wohne ich in Berlin-Kreuzberg; seit mehr als 20 Jahren bin ich in der internationalen Standardisierung engagiert. Während sich unsere Hauptstadt gerne mit dem Attribut „arm aber sexy" schmückt, werden der Normung häufig die entgegen gesetzten Eigenschaften zugesprochen:

" "Standards und Normen sind zwar praktisch aber extrem langweilig",

- "der Standardisierungsprozess ist zäh, bürokratisch und innovationshemmend",

- "Normungsvorhaben dauern viel zu lange und enden dann mit einer Einigung auf den kleinsten gemeinsamen Nenner",

- "Standards sind unzureichend praxisbezogen und nahezu unlesbar",

- "es gibt zu viele Gremien, die zueinander in Konkurrenz stehen und sich gegenseitig behindern".

So oder so ähnlich lauten noch immer die (Vor-)Urteile, die man vielfach zum Thema Standardisierung zu hören bekommt. Dabei sind Standardisierungsprozesse schon lange keine von der Praxis losgelösten, rückwärtsgewandten Gedankenspiele mehr, sondern überaus realitätsbezogen und extrem nützlich. Beispielsweise erbringen Normen einen hohen betriebs- und volkswirtschaftlichen Nutzen, der allein für Deutschland auf jährlich rund 16 Milliarden Euro beziffert wurde'.

Speziell Standards zum Management der Informationssicherheit nach ISO/IEC 27001 haben in den letzten Jahren in ganz erheblichem Maße an Bedeutung und Interesse gewonnen. Ihre große Beliebtheit verwundert nicht, versprechen sie doch u.a. eine Verringerung von Geschäftsrisiken, besser strukturierte Prozesse, Wettbewerbsvorteile, und die Vermeidung von Imageschäden. Edward Humphreys legt den Status Quo der Normung zu diesem Themenkomplex dar, komplementiert durch einen Beitrag von Rainer Rumpel über Erfahrungen aus der Praxis.

Maschinenlesbare Personalausweise oder Reisepässe besitzen wir alle und nutzen die hierdurch gegebenen Erleichterungen beim grenzüberschreitenden Reisen. Die diesen Reisedokumenten und ihren elektronischen Pendants zugrunde liegenden Sicherheitsstandards beleuchte ich in meinem Beitrag.

Beispielhaft für das Aufgreifen neuer Erfordernisse durch Standardisierungsgremien stehen in diesem Heft die viel diskutierten intelligenten Stromnetze (Smart Grid). Sie werden die Möglichkeit bieten, Zustandsinformationen und Lastflussdaten aus einzelnen Netzelementen, wie Erzeugungsanlagen oder Verbrauchsstellen in Echtzeit abrufen und verarbeiten zu können. Datenschutz und Datensicherheit sind hier unverkennbar zentrale Anforderungen ${ }^{2}$ und Mario Wendt diskutiert sowohl die Vielfalt der Gremien als auch den aktuellen Stand der Normungsaktivitäten auf diesem Gebiet.

Eine ebenfalls noch relativ junge Aktivität ist das Herangehen der Standardisierung an das Identitätsmanagent sowie an Technologien zum Schutz personenbezogener Daten, das Kai Rannenberg skizziert. Wertvoll Impulse hierzu kommen aus den Überlegungen für einen modernen, proaktiven Datenschutz von Kirsten Bock und Martin Rost.

Absolut realitätsbezogen und keineswegs auf die Anwendung e-Notar beschränkt ist auch die Forderung nach einer sicheren Langzeitspeicherung von Informationen und einer Langzeitvalidierung von elektronischen Signaturen. Mit diesen Fragestellungen befassen sich die beiden Beiträge von Franco Ruggieri und Gregor Karlinger bzw. Arno Fiedler und Christoph Thiel.

Mit diesem vielfältigen Spektrum an Beiträgen zu aktuellen Normungsthemen hoffen wir den eingangs angesprochenen Vorurteilen wirksam zu begegnen. Abschließend ist festzustellen, dass Standards von der Industrie längst als strategisches Instrument im Wettbewerb erkannt wurden. Vor allem, da durch die Beteiligung an der Normungsarbeit Forschungsrisiken und Entwicklungskosten gesenkt, oder Vorteile durch einen Wissens- und Zeitvorsprung erlangt werden können. Standardisierung, so könnte man folglich sagen, bringt wirtschaftlichen Nutzen und ist „ziemlich sexy".

\section{Walter Fumy}

1 Deutsches Institut für Normung: Gesamtwirtschaftlicher Nutzen der Normung, Zusammenfassung der Ergebnisse - wissenschaftlicher Endbericht mit praktischen Beispielen, Beuth, 2000.

2 Entschließung der 80. Konferenz der Datenschutzbeauftragten in diesem Heft S. 48 